\title{
Drivers of intermittent preventive treatment of malaria during pregnancy in Ghana: a generalized linear model with negative binomial approach
}

\author{
Ernest Yeboah Boateng ${ }^{1}$, Godsway Elorm Anyormi ${ }^{2}$, Joseph Otoo ${ }^{3}$ and Daniel A. Abaye ${ }^{1 *}$ (1)
}

*Correspondence: dabaye@uhas.edu.gh

${ }^{1}$ Department of Basic

Sciences, School of Basic

and Biomedical Sciences,

University of Health

and Allied Sciences, Ho, Volta

Region, Ghana

Full list of author information

is available at the end of the article

\begin{abstract}
Background: This study identified the main drivers of intermittent preventive therapy (IPTp) of malaria in pregnancy with the malaria drugs' sulfadoxine-pyrimethamine (SP) uptake from a longitudinal data set in Ghana.

Method: The data set was obtained from a municipal hospital and spanned a period of 9 years (December 2008-January 2017) and was captured on a monthly basis. We employed the generalized linear modeling approach with negative binomial method to analyze the 9 year data set. A log-linear time-series model was introduced which was then used to validate the results obtained from the logistic model.
\end{abstract}

Results: It is evident from the results that antenatal registration of pregnant women, male partner involvement in antenatal clinic attendance, and frequency of visits to antenatal clinics are the key drivers of IPTP-SP uptake.

Conclusions: We recommend that the Family Planning and the Community Health Nursing units of the Ministry of Health, Ghana, should carry effective health campaigns geared towards raising awareness, promoting the early antenatal registration by pregnant women, encourage male partner involvement in antenatal clinics, and increase the frequency of visits (at least 4) to antenatal clinics by the pregnant women to improve upon the low uptake of IPTp-SP in Ghana.

Keywords: Intermittent preventive treatment, Malaria, Longitudinal data set, Negative binomial model, Sulfadoxine-pyrimethamine, Ghana

\section{Introduction}

Malaria is one of the leading causes of death in many developing nations throughout Africa, South America, and Asia. African countries are most prone to the deadly disease malaria and rising temperatures aid the spread of the disease (WHO 2012). More than 30 million women in Africa become pregnant in malaria endemic areas and are at risk of Plasmodium falciparum malaria infection compared with non-pregnant women. Malaria during pregnancy-related morbidity and mortality is most pronounced in sub-Saharan Africa, yet only a fraction of these women have access to effective anti-malaria interventions (Braun et al. 2015; Gutman and Slutsker 2017).

(c) The Author(s) 2018. This article is distributed under the terms of the Creative Commons Attribution 4.0 International License (http://creativecommons.org/licenses/by/4.0/), which permits unrestricted use, distribution, and reproduction in any medium, provided you give appropriate credit to the original author(s) and the source, provide a link to the Creative Commons license, and indicate if changes were made. 
In Ghana, malaria among pregnant women accounts for about $14 \%$ of Out Patient Department (OPD) attendance, 11\% of admissions, and 9\% of deaths (Odjidja et al. 2017).

Malaria infections cause a tremendous burden on the health systems in these countries and primarily affect pregnant women (WHO 2003). Malaria during pregnancy results in adverse birth outcomes and poor maternal health (WHO 2014). When the mother is infected during pregnancy, the parasite is passed along with the fetus. This event results in stillbirths and low birth weight which is the single greatest risk factor for neonatal deaths. A rapid and severe case of malaria during pregnancy can also weaken the immune system, which cause maternal and fetal anemia, seizures, coma, or respiratory infection, all of which lead to death (Dellicour et al. 2010).

Consequently, the WHO recommends protection for women during pregnancy. Until recently, prevention consisted of weekly chemoprophylaxis with either chloroquine or sulfadoxine-pyrimethamine (SP) (WHO 2004). Because of poor patient compliance with prophylaxis and increasing resistance of parasite strains to chloroquine, administration of intermittent preventive treatment in pregnancy (IPTp) with SP (ITPp-SP) is now recommended for all pregnant women living in areas with stable to high malaria transmission (Braun et al. 2015). SP is given during antenatal visits at curative doses (1500 $\mathrm{mg}$ sulfadoxine and $75 \mathrm{mg}$ pyrimethamine; that is, $3 \times$ the prophylactic dosage previously used) at least twice during pregnancy, once at the second trimester and once at least 1 month after the first treatment (Ibrahim et al. 2017).

Following the recommendation of the WHO in 2000, Ghana adopted a new malaria treatment policy in 2004 which was revised in 2007, and, again, in 2012. The country, thus, moved from the use of mono-therapy to combination therapy using artemisininbased combination therapy (ACT) (Plan 2014). Part of this policy was the change from use of weekly chloroquine chemoprophylaxis to SP as intermittent for malaria prevention during pregnancy (WHO 2014). The recommended interventions for malaria in pregnancy consist of three tenets; intermittent preventive treatment (IPT), insecticidetreated bed nets (ITNs), and appropriate case management of malaria illness (Desai et al. 2007).

Besides their clinical efficacy, the effectiveness of these interventions depends on the attitudes and behaviours of pregnant women and the wider community, which are shaped by social and cultural factors. Attitudes towards and understandings of pregnancy, pregnancy care, malaria, and other illnesses can interact and influence how, where, and when pregnant women seek malaria prevention and treatment (Dhiman et al. 2012). Furthermore, the social and cultural context has important implications for the provision of malaria prevention or treatment, whether as part of routine ANC at a health facility or care sought from a local healer (Dellicour et al. 2010).

The study by Dhiman et al. (2012) showed that women's limited knowledge about the health consequences of malaria during pregnancy, antenatal clinic (ANC) health workers' lack of familiarity with IPTp, and the association of sleeping under a bed net when pregnant or receiving IPTp with education and/or wealth were significant predictors of IPTp-SP uptake. Ibrahim et al. (2017) demonstrated that having a good knowledge of malaria in pregnancy and IPT of malaria in pregnancy can significantly influence the uptake of optimal doses of SP. In addition, encouraging women to attend 
antenatal care regularly (at least four visits) could also increase the optimal uptake of sulfadoxine-pyrimethamine.

Pell et al. (2011) revealed understandings of ANC, health worker-client interactions, household decision-making, gender relations, cost, and distance to health facilities affect pregnant women's access to malaria in pregnancy interventions, and lack of healthcare infrastructure limits the uptake of IPTp-SP. The number of visits to antenatal clinics, governance, finance, product and technology, health workforce, health information, and service delivery were underlying factors identified as affecting optimal uptake of IPTp-SP in Ghana (Odjidja et al. 2017).

In determining the level of uptake of SP as baseline for monitoring progress and reviewing stock levels of SP, Owusu-Boateng and Anto (2017) carried out a cross-sectional hospital-based study among nursing mothers who had delivered within 12 weeks and seeking postnatal care at the Osu Government Maternity Home in Accra, Ghana. The results of this study indicated that the proportions of uptake of the three-to-five doses of SP were: IPTp3 (87.5\%), IPTp4 (55.7\%), and IPTp5 (14.5\%), even though the stock levels of SP were adequate to meet the needs by the pregnant women at the Maternity Home for the period under review.

Elsewhere in sub-Saharan Africa, IPTp-SP uptake is affected by stock-out and nonadherence to protocols by health care providers (Plan 2014). Several other servicerelated and community factors, such as unavailability of skilled attendants at ANC, low or lack of male partner involvement in antenatal clinic (Yargawa and Leonardi-Bee 2015), poor attitudes of clinic staff, and pregnant women not registering at antenatal clinics, hinder the implementation of IPTp-SP (ter Kuile et al. 2007). In some cases, the women are given the drug, and yet, they do not swallow it as it is not always given as directly observed therapy (Plan 2014).

Braun et al. (2015) performed systematic review and meta-analysis of trials to determine whether regimens containing three or more doses of SP for intermittent preventive therapy during pregnancy are associated with a higher birth weight or lower risk of low birth weight $(\mathrm{LBW})(<2500 \mathrm{~g})$ than standard two-dose regimens. Results indicated that three or more doses were associated with fewer LBW births and the $\geq 3$-dose group had less placental malaria; however, there were no differences in rates of serious adverse events.

Although these factors have been studied largely using qualitative methods, quantitative research also offers important insights. Ghana has adopted WHO recommendations on the use of SP for IPTp since 2004, but coverage still remains generally low. Like many other endemic countries, Ghana's ability to achieve the global target of $80 \%$ IPT coverage still poses a great challenge (Yargawa and Leonardi-Bee 2015). Data on knowledge and factors enhancing IPT uptake have generally been collected using (more) cross-sectional quantitative methods, such as questionnaires, with comparatively little research based on longitudinal data using quantitative methods. However, quantitative research that situates behaviour in its context is also necessary to give insight into findings highlighted by qualitative research (Gutman and Slutsker 2017).

In as much as a significant number of studies have been carried out on IPTp-SP uptake, a majority of these studies (e.g. Desai et al. 2007; ter Kuile et al. 2007; Gutman and Slutsker 2017; Owusu-Boateng and Anto 2017; Odjidja et al. 2017; Yargawa and 
Leonardi-Bee 2015) are cross-sectional with two (Dellicour et al. 2010; Dhiman et al. 2012) being meta-analysis. Specifically, in Ghana, IPTp-SP studies which carried out have all been cross-sectional with results indicating low uptake (Owusu-Boateng and Anto 2017; Odjidja et al. 2017).

\section{Aims}

In the light of the issues affecting the uptake of IPTp in Ghana espoused in the foregoing paragraphs, there is the need to identify the key drivers of IPTp-SP uptake. This paper explores the factors that influence IPTp-SP uptake from a longitudinal data set. The novelty of our work is that the study employs the generalized linear modeling approach to elucidate the drivers of IPTp-SP uptake in Ghana. Our study thus presents a statistical enquiry to elucidate the potential main drivers of IPTp-SP uptake in Ghana utilizing a longitudinal data set.

\section{Materials and methods}

Data

Data for the study are a longitudinal count data obtained from the Sunyani Municipal Hospital in the Brong Ahafo Region of Ghana. The hospital serves approximately 150,000 residents.

Count data generally are numbers of events per interval (Venables and Ripley 2002). Mathematically, counts are non-negative integers, since an event cannot happen an incomplete or a negative number of times, and essentially, there is no upper boundary to a count, because there can theoretically be close to an infinite number of events taking place.

The longitudinal data set comprises vital maternal variables of interest such as antenatal clinic registration of pregnant women, IPTp uptake, age of pregnant women, family planning, number of visits at the clinic, number of pregnancies and births by pregnant women, distance from their homes to the hospital, male partner attendance at clinic which were recorded over the period of 9 years (from December 2008 to January 2017). These events were recorded on a monthly basis.

\section{The negative binomial method}

The negative binomial method which is an extension of the generalized linear models was used in analyzing the longitudinal data set. This method was chosen, because the counts or values recorded for the IPT dosage (IPTp 1-IPTp 5) were over-dispersed (i.e., variance is greater than the mean). The over-dispersion is detailed in Table 1.

The distribution of negative binomial model for the over-dispersed count data is expressed as follows:

$$
\operatorname{Pr}(Y=y \mid \lambda, \alpha)=\frac{\Gamma\left(y+\alpha^{-1}\right)}{y ! \Gamma\left(\alpha^{-1}\right)}\left(\frac{\alpha^{-1}}{\alpha^{-1}+\lambda}\right)^{\alpha^{-1}}\left(\frac{\lambda}{\alpha^{-1}+\lambda}\right)^{y},
$$

where $\lambda$ is the mean value or the expected value of the distribution and $\alpha$ is the overdispersion parameter.

The negative binomial model (log transformed) for the study is, therefore: 
Table 1 Table of means and variances of IPTP-SP

\begin{tabular}{lrccl}
\hline IPT & Mean & Variance & Ratio (var/mean) & Remark \\
\hline IPT1 & 104.31 & 784.79 & 7.52 & Over-dispersed \\
IPT2 & 90.09 & 602.77 & 6.69 & Over-dispersed \\
IPT3 & 70.15 & 473.80 & 6.75 & Over-dispersed \\
IPT4 & 9.40 & 254.40 & 27.04 & Over-dispersed \\
IPT5 & 3.01 & 36.01 & 11.96 & Over-dispersed \\
\hline
\end{tabular}

$$
\begin{aligned}
\log \mathrm{IPT}_{i}=\beta_{0} & +\beta_{1} \mathrm{ANC}_{\mathrm{REG}}+\beta_{2} \mathrm{M}_{\mathrm{ANC}}+\beta_{3} \mathrm{ANC}_{\mathrm{V} 1}+\beta_{4} \mathrm{ANC}_{\mathrm{V} 2}+\beta_{5} \mathrm{ANC}_{\mathrm{V} 4}+\beta_{6} \mathrm{~B}_{1519} \\
& +\beta_{7} \mathrm{~B}_{20_{24}}+\beta_{8} \mathrm{~B}_{25_{29}}+\beta_{9} \mathrm{~B}_{30_{34}}+\beta_{10} \mathrm{~B}_{35}+\beta_{11} \mathrm{M} \_ \text {PARA }+\beta_{12} \mathrm{P} \_\mathrm{PARA},
\end{aligned}
$$

for $i=1,2, \ldots, 5$.

Where $\mathrm{IPT}_{i}=$ intermittent preventive treatment 1 through to $5 ; \mathrm{ANC}_{\mathrm{REG}}=$ antenatal clinic registration of pregnant women; $M_{A N C}=$ male involvement at antenatal clinic; $\mathrm{B}_{15_{19}}=$ pregnant women of age $15-19$ who received IPTp; $\mathrm{B}_{20_{24}}=$ pregnant women of age 20-24 who received IPTp; $B_{25_{29}}=$ pregnant women of age 25-29 who received IPTp; $\mathrm{B}_{30_{34}}=$ pregnant women of age $30-34$ who received IPTp; $\mathrm{B}_{35}=$ pregnant women of age $\geq 35$ years who received IPTp; $\mathrm{ANC}_{\mathrm{V} 1}=$ one visit to the antenatal clinics by pregnant women; $\mathrm{ANC}_{\mathrm{V} 2}=$ two visits to the antenatal clinics by pregnant women; $\mathrm{ANC}_{\mathrm{V} 4}=$ four visits to the antenatal clinics by pregnant women; M_PARA = four and greater number of births; P_PARA = first birth of a woman.

\section{The log-linear time-series model}

We introduced a log-linear time-series model, which was then used to validate the results obtained from the logistic model. The general form of the model is given as follows:

$$
v_{t}=\beta_{0}+\sum_{k=1}^{p} \beta_{k} \log \left(Y_{t-k}+1\right)+\sum_{\ell=1}^{q} \alpha_{\ell} v_{t-\ell}
$$

The model for this study was formulated as follows:

$$
\begin{aligned}
\log \left(\mathrm{IPT}_{i}\right) & =\beta_{0}+\sum_{k=1}^{5} \beta_{k} \log \left(\mathrm{IPT}_{i t-k}+1\right)+\eta_{1} \log \left(\mathrm{ANC}_{\mathrm{REG}}\right)+\eta_{2} \log \left(\mathrm{ANC}_{\mathrm{V} 1}\right) \\
& +\eta_{3} \log \left(\mathrm{ANC}_{\mathrm{V} 2}\right)+\eta_{4} \log \left(\mathrm{ANC}_{\mathrm{V} 4}\right)+\eta_{5} \log \left(\mathrm{M}_{\mathrm{ANC}}\right)+\mathrm{LT}_{t}
\end{aligned}
$$

for $i=1,2,3$.

Where $\mathrm{LT}_{t}$ is the linear trend at time $t ; \sum_{k=1}^{5} \beta_{k} \log \left(\mathrm{IPT}_{i t-k}+1\right)$ is the autoregressive term of IPT; $\eta_{1}, \eta_{2}, \ldots, \eta_{5}$ are the coefficients of the covariates; $\sum_{\ell=1}^{q} \alpha_{\ell} v_{t-\ell}$ is the summation of the covariates.

\section{Analysis}

In building the negative binomial models, the IPTs were used as the response variables for the three separate models. For the independent variables, antenatal clinic 
registration, male involvement at antenatal clinic, number of visits to the antenatal clinics, age group at which women give birth, and number of children ever born by women were used.

Specifically, the response variables (IPTs) for the three separate models were captured in the respective models as IPT1 for the first model, IPT2 for the second model, and IPT3 for the third model. For the independent variables, antenatal clinic registration was captured in the model as $\mathrm{ANC}_{\mathrm{REG}}$; male involvement at antenatal clinic as $\mathrm{M}_{\mathrm{ANC}}$; number of visits to the antenatal clinics as $\mathrm{ANC}_{\mathrm{V} 1}, \mathrm{ANC}_{\mathrm{V} 2}$, and $\mathrm{ANC}_{\mathrm{V} 4}$; age group at which women give birth as $\mathrm{B}_{15_{19}}, \mathrm{~B}_{20_{24}}, \mathrm{~B}_{25_{29}}, \mathrm{~B}_{30_{34}}$, and $\mathrm{B}_{35}$; number of children ever born by women as M_PARA and P_PARA. The R Statistical software (Team 2015) with it packages such as MASS and Zoo were used for the analyses.

\section{Results}

Figure 1 shows the monthly time-series trend of dosage administered to pregnant women and the monthly ANC registrations from 2008 to 2017. The trend indicates that ANC registration, IPT1, IPT2, and IPT3, had rising trends for the period of 2008-2014. However, beyond 2014 to 2017, there was steady decline in ANC registration as well as $\mathrm{IPT}_{\mathrm{P}}$ uptake. This result indicates that the uptake of IPT $\mathrm{P}_{\mathrm{P}}$ increases as ANC registration also increases. The IPT4 and IPT5 were not included in the modeling due to the fact that their observed data points were few (3 years). We hold the view that 3 years of observation are not enough to extract the drivers IPTp-SP from the data.

\section{Binomial logistic regression model}

The respective means, variances, and ratios of variance to mean of IPT1-IPT5 with their corresponding remarks are given in Table 1. The over-dispersion remark for IPT1-IPT5 signifies that the Poisson model which is based on equal mean and variance cannot fit the data for the study (data available upon request). In this regard, the negative binomial model which is based on over-dispersion is adopted.

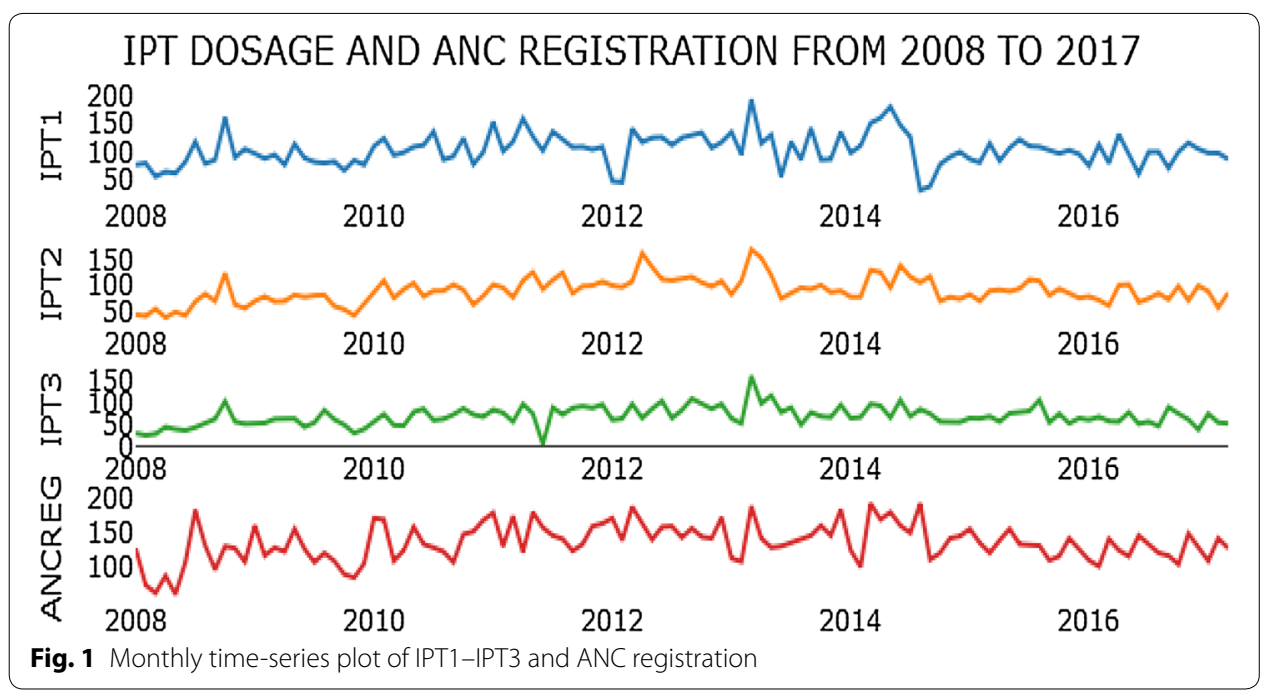


Table 2 Results of negative binomial model (model 1)

\begin{tabular}{lclcc}
\hline IPT factor & Estimate & Standard error & $\boldsymbol{Z}$ value & $P$ value \\
\hline Intercept & 56.530 & 0.120 & 33.450 & $0.000^{*}$ \\
$\mathrm{ANC}_{\mathrm{REG}}$ & 1.004 & 0.000 & 5.099 & $0.000^{*}$ \\
$\mathrm{~B}_{15} 5_{19}$ & 1.005 & 0.004 & 0.990 & 0.322 \\
$\mathrm{~B}_{25}$ & 0.996 & 0.002 & -1.320 & 0.187 \\
$\mathrm{~B}_{30} 0_{34}$ & 1.000 & 0.003 & 0.162 & 0.871 \\
$\mathrm{~B}_{35}$ & 0.996 & 0.004 & -0.744 & 0.457 \\
$\mathrm{M}_{\mathrm{ANC}}$ & 1.001 & 0.002 & 0.647 & 0.518 \\
$\mathrm{ANC}_{\mathrm{V} 1}$ & 1.000 & 0.000 & 0.885 & 0.376 \\
$\mathrm{ANC}_{\mathrm{V} 2}$ & 1.000 & 0.000 & 0.745 & 0.456 \\
$\mathrm{ANC}_{\mathrm{V} 4}$ & 1.000 & 0.000 & 1.587 & 0.113 \\
\hline
\end{tabular}

*Figures in italics are significant $(P<0.05)$

Table 3 Results of negative binomial model (model 2)

\begin{tabular}{lclcc}
\hline IPT Factor & Estimate & Standard error & $\boldsymbol{Z}$ value & $P$ value \\
\hline Intercept & 40.540 & 0.110 & 33.450 & $0.000^{*}$ \\
$\mathrm{ANC}_{\mathrm{REG}}$ & 1.005 & 0.001 & 6.385 & $0.000^{*}$ \\
$\mathrm{~B}_{15} 5_{19}$ & 0.997 & 0.004 & -0.731 & 0.465 \\
$\mathrm{~B}_{20} 0_{24}$ & 0.998 & 0.003 & -0.858 & 0.391 \\
$\mathrm{~B}_{30}$ & 1.005 & 0.003 & 1.570 & 0.114 \\
$\mathrm{~B}_{35}$ & 0.999 & 0.004 & -0.315 & 0.753 \\
$\mathrm{M}_{\mathrm{ANC}}$ & 1.005 & 0.002 & 2.244 & $0.025^{*}$ \\
$\mathrm{ANC}_{\mathrm{V} 1}$ & 1.000 & 0.000 & 1.080 & 0.280 \\
$\mathrm{ANC}_{\mathrm{V} 2}$ & 0.999 & 0.000 & -1.203 & 0.220 \\
$\mathrm{ANC}_{\mathrm{V} 4}$ & 1.001 & 0.000 & 2.693 & $0.007^{*}$ \\
\hline
\end{tabular}

*Figures in italics are significant $(P<0.05)$

Table 4 Results of negative binomial model (model 3)

\begin{tabular}{lclcc}
\hline IPT factor & Estimate & Standard error & $\boldsymbol{Z}$ value & $\boldsymbol{P}$ value \\
\hline Intercept & 36.690 & 0.138 & 25.698 & $0.000^{*}$ \\
$\mathrm{ANC}_{\mathrm{REG}}$ & 1.004 & 0.000 & 4.235 & $0.000^{*}$ \\
$\mathrm{~B}_{15}{ }_{19}$ & 1.006 & 0.005 & 1.018 & 0.308 \\
$\mathrm{~B}_{25}$ & 0.999 & 0.003 & -0.304 & 0.761 \\
$\mathrm{~B}_{30} 0_{34}$ & 0.999 & 0.003 & -0.135 & 0.892 \\
$B_{35}$ & 0.999 & 0.005 & -0.091 & 0.927 \\
$M_{A N C}$ & 1.007 & 0.003 & 2.481 & $0.013^{*}$ \\
ANC $_{\mathrm{V} 1}$ & 1.000 & 0.000 & 1.572 & 0.115 \\
$\mathrm{ANC}_{\mathrm{V} 2}$ & 0.998 & 0.000 & -2.092 & $0.036^{*}$ \\
$\mathrm{ANC}_{\mathrm{V} 4}$ & 1.001 & 0.000 & 2.772 & $0.005^{*}$ \\
\hline
\end{tabular}

*Figures in italics are significant $(P<0.05)$

The results of the analyses of the longitudinal data set are detailed in Tables 2, 3, 4, and 5, respectively. Results from the negative binomial model indicate that antenatal registration was statistically significant $(P<0.05)$ to uptake of IPT1. The results (Table 2 , model 1) show that a unit increase in the proportion of pregnant women who register at 


Table $\mathbf{5}$ Chi-square $\left(\mathbf{X}^{\mathbf{2}}\right)$ test of goodness of fit based on the residual deviance and degrees
of freedom
\begin{tabular}{llll}
\hline Model & Significant variables & $\left(\boldsymbol{X}^{\mathbf{2}}, \boldsymbol{P}\right.$ value $)$ & Implication \\
\hline 1 & Intercept and $A N C_{\mathrm{REG}}$ & 0.184 & Good fit of data \\
2 & Intercept, $A N C_{\mathrm{REG}}, \mathrm{M}_{\mathrm{ANC}}$ and $A N C_{\mathrm{V} 4}$ & 0.208 & Good fit of data \\
3 & Intercept, $A N C_{\mathrm{REG}}, \mathrm{M}_{\mathrm{ANC}}, \mathrm{ANC}_{\mathrm{V} 2}$ and $A N C_{\mathrm{V} 4}$ & 0.124 & Good fit of data
\end{tabular}

antenatal clinic leads to the corresponding increase in the uptake of IPT1 for pregnant women by 1.004 , holding the other variables constant. In other words, registering one additional pregnant woman at the antenatal clinic will increase the uptake of IPT1 for pregnant women by $0.4 \%$, with the other variables constant.

The exponentiated values of the model are captured by the second column on Table 2 . From Table 2, the intercept and $\mathrm{ANC}_{\mathrm{REG}}$ are statistically significant with the corresponding $P$ values 0.00 and 0.00 , respectively. The implication of this result is that the other variables $P$ value $>0.05$ are statistically insignificant to uptake of IPT1.

Results from Table 3 (model 2 ) indicate antenatal registration $\left(\mathrm{ANC}_{\mathrm{REG}}\right)$, male involvement at antenatal clinic $\left(\mathrm{M}_{\mathrm{ANC}}\right)$, and the fourth visit $\left(\mathrm{ANC}_{\mathrm{V} 4}\right)$ to the antenatal clinic which were statistically significant $(P<0.05)$ to uptake of IPT2. The results as shown in Table 3 imply that a unit increase in the proportion of pregnant women who register at antenatal clinic corresponds to increase of the uptake of IPT2 for pregnant women by 1.005 holding the other variables constant. In addition, a unit increase in the proportion of men involved at antenatal clinic corresponds to increase of the uptake of IPT2 for pregnant women by 1.005 holding the other variables constant. Finally, an increase in the number of the fourth visits to antenatal clinic by pregnant women corresponds to increase of the uptake of IPT2 for pregnant women by 1.001, with the other variables constant.

The implications are that an increase in the proportion of pregnant women who register at antenatal clinics, male involvements at the antenatal clinic, and the number of visits to the antenatal clinic yield the corresponding increase of the uptake of IPT 2 by $0.5 \%$, $0.5 \%$, and $0.1 \%$, respectively, with the other variables constant.

The exponentiated values of the model are captured by the second column on the table. From the table, the intercept, $\mathrm{ANC}_{\mathrm{REG}}, \mathrm{M}_{\mathrm{ANC}}$, and $\mathrm{ANC}_{\mathrm{V} 4}$ are statistically significant with the corresponding $P$ values $0.00,0.00,0.02$, and 0.00 , respectively. The implication of this result is that the other variables with $P$ values $>0.05$ are statistically insignificant to uptake of IPT2.

Results detailed in Table 4 (model 3) revealed that antenatal registration $\left(\mathrm{ANC}_{\mathrm{REG}}\right)$, male involvement $(\mathrm{ANC} \mathrm{M})$ at antenatal clinic, and the second $\left(\mathrm{ANC}_{\mathrm{V} 2}\right)$ and fourth $\left(\mathrm{ANC}_{\mathrm{V} 4}\right)$ visits to the antenatal clinic by pregnant women were statistically significant $(P<0.05)$ to uptake of IPT3. The results imply that a unit increase in the proportion of pregnant women who register at antenatal clinic corresponds to increase of the uptake of IPT3 for pregnant women by 1.004 holding the other variables constant. A unit increase in the proportion of men involved at antenatal clinic corresponds to increase of the uptake of IPT3 for pregnant women by 1.007 holding the other variables constant. An increase in the number of the second visits to antenatal clinic by pregnant 
Table 6 Results of log-linear time-series models (model 4) for IPT1

\begin{tabular}{lrlcl}
\hline IPT factor & Estimate & Standard error & Cl (lower) & Cl (upper) \\
\hline Intercept & $3.09 e+00$ & 0.473235 & 2.411866 & $4.164283^{*}$ \\
$\mathrm{IPT}_{1 t-1}$ & $1.83 e-01$ & 0.081848 & -0.001950 & 0.309911 \\
$\mathrm{IPT}_{1 t-5}$ & $2.06 \mathrm{e}-02$ & 0.082073 & -0.149237 & 0.164093 \\
$\mathrm{ANC}_{\mathrm{V} 1}$ & $-5.38 \mathrm{e}-05$ & 0.000206 & -0.000469 & 0.000293 \\
$\mathrm{ANC}_{\mathrm{V} 2}$ & $5.42 \mathrm{e}-04$ & 0.000436 & -0.000262 & 0.001496 \\
$\mathrm{ANC}_{\mathrm{V}}$ & $9.73 e-05$ & 0.000271 & -0.000441 & 0.000609 \\
$\mathrm{ANC}_{\text {REG }}$ & $3.72 e-03$ & 0.000878 & 0.002165 & $0.005508^{*}$ \\
$\mathrm{M}_{\text {ANC }}$ & $1.10 \mathrm{e}-03$ & 0.002509 & -0.004196 & 0.005509 \\
$\mathrm{LT}_{t}$ & $9.17 \mathrm{e}-03$ & 0.013976 & -0.016773 & 0.039059 \\
\hline
\end{tabular}

*Figures in italics are significant $(P<0.05)$ (if the confidence interval, $\mathrm{Cl}$, does not include zero)

women corresponds to a reduction or decrease of the uptake of IPT3 for pregnant women by 0.002 holding the other variables constant. Finally, an increase in the number of the fourth visits to antenatal clinic by pregnant women corresponds to increase of the uptake of IPT3 for pregnant women by 1.001 holding the other variables constant.

The implications are that an increase in the proportion of pregnant women who register at antenatal clinic, male involvement at the antenatal clinic, and fourth number of visits to the antenatal clinic yield the corresponding increase of the uptake of IPT3 by $0.4 \%, 0.7 \%$, and $0.1 \%$, respectively, holding the other variables constant. In addition, an increase in the number of the second visits to antenatal clinic by pregnant women yields a reduction or decrease of the uptake of IPT3 for pregnant women by $0.2 \%$, with the other variables constant.

The exponentiated values of model 3 are captured by the second column (estimate) of Table 4. From the table, the intercept, $\mathrm{ANC}_{\mathrm{REG}}, \mathrm{M}_{\mathrm{ANC}}, \mathrm{ANC}_{\mathrm{V} 2}$, and $\mathrm{ANC}_{\mathrm{V} 4}$ are statistically significant $(P<0.05)$ with the corresponding $P$ values $0.00,0.00,0.013$, and 0.005 , respectively. The implication of this result is that the other variables with $P$ values $>0.05$ are statistically insignificant to uptake of IPT3.

The Chi-square test of goodness of fit based on the residual deviance and degrees of freedom was performed. The goodness-of-fit test indicates that the negative binomial models (model 1 , model 2 , and model 3$)$ fit the data $(P>0.05)$ (Table 5$)$.

\section{The log-linear time-series model}

Tables 6, 7, 8 capture the results for the log-linear time-series models for the uptake of IPT1, IPT2, and IPT3, respectively. The results from the models confirm the results obtained by the negative logistic binomial models. Although the $P$ value is generally used to test the significance of variables in models, the confidence interval was used for the log-linear time-series model. Using the confidence interval approach, the null hypothesis is accepted to be true if the confidence interval includes zero. The significant variables in the model have been highlighted. 
Table 7 Results of log-linear time-series models (model 5) for IPT2

\begin{tabular}{lclcl}
\hline IPT factor & Estimate & Standard error & Cl (lower) & Cl (upper) \\
\hline Intercept & 1.594638 & 0.316685 & 1.188432 & $2.438315^{*}$ \\
$\mathrm{IPT}_{2 t-1}$ & 0.422851 & 0.073885 & -0.235606 & 0.529580 \\
$\mathrm{IPT}_{2 t-5}$ & 0.120454 & 0.071273 & -0.035429 & 0.229242 \\
$\mathrm{ANC}_{\mathrm{V} 1}$ & -0.000196 & 0.000198 & -0.000582 & 0.000185 \\
$\mathrm{ANC}_{\mathrm{V} 2}$ & 0.000406 & 0.000409 & -0.000424 & 0.001189 \\
$\mathrm{ANC}_{\mathrm{V}}$ & 0.0164 & 0.00236 & 0.00618 & $0.0293^{*}$ \\
$\mathrm{ANC}_{\text {REG }}$ & 0.003249 & 0.000730 & 0.002037 & $0.004738^{*}$ \\
$\mathrm{M}_{\text {ANC }}$ & 0.003724 & 0.001994 & 0.001035 & $0.006569^{*}$ \\
$\mathrm{LT}_{t}$ & -0.002544 & 0.012585 & -0.026671 & 0.024447 \\
\hline
\end{tabular}

*Figures in italics are significant $(P<0.05)$ (if the confidence interval, $\mathrm{Cl}$, does not include zero)

Table 8 Results of log-linear time-series models (model 6) for IPT3

\begin{tabular}{lclcl}
\hline IPT factor & Estimate & Standard error & Cl (lower) & Cl (upper) \\
\hline Intercept & 2.758043 & 0.491316 & 1.985361 & $3.883321^{*}$ \\
$\mathrm{IPT}_{3 t-1}$ & 0.115501 & 0.090893 & -0.087948 & 0.262227 \\
$\mathrm{IPT}_{3 t-5}$ & 0.096229 & 0.088969 & -0.097480 & 0.240913 \\
$\mathrm{ANC}_{\mathrm{V} 1}$ & 0.000043 & 0.000263 & -0.000531 & 0.000477 \\
$\mathrm{ANC}_{\mathrm{V} 2}$ & 0.00112 & 0.00562 & 0.000976 & $0.003564^{*}$ \\
$\mathrm{ANC}_{\mathrm{V} 4}$ & 0.00556 & 0.0333 & 0.001223 & $0.049^{*}$ \\
$\mathrm{ANC}_{\text {REG }}$ & 0.004483 & 0.001050 & 0.002569 & $0.006657^{*}$ \\
$\mathrm{M}_{\text {ANC }}$ & 0.03078 & 0.003088 & 0.003939 & $0.08907^{*}$ \\
$\mathrm{LT}_{t}$ & -0.001705 & 0.016936 & -0.031371 & 0.032214 \\
\hline
\end{tabular}

*Figures in italics are significant $(P<0.05)$ (if the confidence interval, $\mathrm{Cl}$, does not include zero)

\section{Discussion}

Employing the negative binomial logistic and the log-linear time-series models, the study demonstrated clearly that the most significant factors influencing the uptake of IPTp are as follows: pregnant women antenatal clinic registration, male partner involvement at the clinic, and number of visits of the pregnant women at the clinics.

Antenatal clinic registration of pregnant women plays a crucial role in the uptake of IPT1, 2, and IPT3. It is also evident from the results of the six models in this study that antenatal clinic registration was statistically significant with a corresponding average increment of $0.4 \%$ on the uptake of IPTs. This confirms results obtained by Ibrahim et al. (2017) and ter Kuile et al. (2007) that pregnant women not registering at antenatal clinics and poor attitudes of health services staff still hinder the implementation of IPTp-SP. Ghana's low uptake (38.5\%) of IPTp-SP Odjidja et al. (2017) could, therefore, be scaled up by introducing interventions and measures that will ensure increasing number of pregnant women register at the antenatal clinics.

In developing countries such as Ghana, empirical studies such as Yargawa and Leonardi-Bee (2015) revealed that male involvement in maternal issues is associated with improved maternal health outcomes. In this present study, results from two out of three models confirmed this outcome. As evidenced by the results of the present study, increment in the proportion of men involved in antenatal clinic increases uptake of IPT 2 and IPT3 by $0.5 \%$ and $0.7 \%$, respectively. 
To scale up Ghana's $38.5 \%$ low uptake of IPTp-SP, the number of visits to the antenatal clinic by pregnant women must be frequent and be at least four, ideally $>4$ visits. Results of this study reveal that number of visits less than four $(<4$ visits $)$ reduced the uptake of IPT3 for pregnant women by $0.2 \%$. This also confirms the findings of Odjidja et al. (2017) who asserted that a number of visits to antenatal clinics, in addition to other factors, including governance, finance, product, technology, health workforce, health information, and service delivery, were underlying the factors affecting optimal uptake of IPTp-SP in Ghana.

\section{Conclusions}

The study employed the negative binomial logistic and the log-linear time-series models to elucidate the drivers of IPTp-SP uptake in Ghana from a longitudinal data set. It is evident from models 1 to 6, whose results are displayed in Tables 2, 3, 4, 5, 6, 7 and 8 , respectively, that antenatal registration, male involvement in antenatal clinic attendance, and the number of visits to antenatal clinic were identified to be the main drivers of uptake of IPTp-SP.

In the light of the results revealed in this study, it is recommended that the Family Planning and the Community Health Nursing Units of the Ministry of Health should be adequately and efficiently resourced to carry effective health campaigns geared towards encouraging the early antenatal registration by pregnant women, male involvement in antenatal clinics, and increasing the frequency of visits (a minimum of 4) to antenatal clinics by the pregnant women. This will aid in scaling up the low uptake of IPTp-SP in Ghana.

\section{Abbreviations}

ANC: antenatal care; ITNs: insecticide-treated nets; IPTp: intermittent preventive therapy; IPTp-SP: intermittent preventive therapy with the drugs sulfadoxine-pyrimethamine.

Authors' contributions

EYB, GEA, and JO developed the idea. Data were collected and analyzed by EYB, GEA, JO, and DAA. Manuscript writing was led by EYB and DAA. All authors read and approved the final manuscript.

\section{Author details}

${ }^{1}$ Department of Basic Sciences, School of Basic and Biomedical Sciences, University of Health and Allied Sciences, Ho, Volta Region, Ghana. ${ }^{2}$ Institute of Distance Learning, University of Ghana, Legon, Accra, Ghana. ${ }^{3}$ Department of Statistics, University of Ghana, Legon, Accra, Ghana.

\section{Acknowledgements}

The authors would like to thank Sunyani Municipal Hospital, Sunyani, Ghana, for allowing access to the secondary data.

Competing interests

The authors declare that they have no competing interests regarding the study and publication of this manuscript.

Availability of data and materials

The data for this study are readily available upon request.

Funding

There was no funding for this study. This manuscript was part of our primary work for all involved.

\section{Publisher's Note}

Springer Nature remains neutral with regard to jurisdictional claims in published maps and institutional affiliations. 
References

Braun V, Rempis E, Schnack A, Decker S, Rubaihayo J, Tumwesigye NM, Mockenhaupt FP (2015) Lack of effect of intermittent preventive treatment for malaria in pregnancy and intense drug resistance in western Uganda. Malar J 14(1):372. https:// doi.org/10.1186/s12936-015-0909-7

Dellicour S, Tatem AJ, Guerra CA, Snow RW, ter Kuile FO (2010) Quantifying the number of pregnancies at risk of malaria in 2007: a demographic study. PLoS Med 7(1):e1000221. https://doi.org/10.1371/journal.pmed.1000221

Desai M, ter Kuile FO, Nosten F, McGready R., Asamoa K, Brabin B, Newman RD (2007) Epidemiology and burden of malaria in pregnancy. Lancet Infect Dis 7(2):93-104. https://www.researchgate.net/publication/6552754_Epidemiology_and_ burden_of_malaria_in_pregnancy

Dhiman S, Yadav K, Goswami D, Das NG, Baruah I, Singh L (2012) Epidemiology and risk analysis of malaria among pregnant women. Iran J Public Health 41(1):1

Gutman J, Slutsker L (2017) Intermittent preventive treatment with sulfadoxine-pyrimethamine: more than just an antimalarial? Am J Trop Med Hyg 96(1):9-10

Ibrahim H, Maya ET, Issah K, Apanga PA, Bachan EG, Noora CL (2017) Factors influencing uptake of intermittent preventive treatment of malaria in pregnancy using sulphadoxine pyrimethamine in Sunyani Municipality, Ghana. Pan Afr Med J 28:122

Odjidja EN, Kwanin C, Saha M (2017) Low uptake of intermittent preventive treatment in Ghana: an examination of health system bottlenecks. Health Syst Policy Res 4(3). http://www.hsprj.com/health-maintanance/low-uptake-of-intermitte nt-preventive-treatment-in-ghana-an-examination-of-health-system-bottlenecks.php?aid=20149

Owusu-Boateng I, Anto F (2017) Intermittent preventive treatment of malaria in pregnancy: a cross-sectional survey to assess uptake of the new sulfadoxine-pyrimethamine five dose policy in Ghana. Malar J 16(1):323. https://doi.org/10.1186/ s12936-017-1969-7

Pell C, Straus L, Andrew EV, Meñaca A, Pool R (2011) Social and cultural factors affecting uptake of interventions for malaria in pregnancy in Africa: a systematic review of the qualitative research. PLOS ONE 6(7):e22452

Plan EL (2014) Modeling and simulation of count data. CPT Pharmacomet Syst Pharmacol 3(8):1-12. https://doi.org/10.1038/ psp.2014.27

Team RC (2015) R: a language and environment for statistical computing. Vienna, Austria: R Foundation for Statistical Computing; 2014. http://www.R-project.org

ter Kuile FO, van Eijk AM, Filler SJ (2007) Effect of sulfadoxine-pyrimethamine resistance on the efficacy of intermittent preventive therapy for malaria control during pregnancy: a systematic review. JAMA 297(23):2603-2616. https://pdfs.seman ticscholar.org/e405/03ab57bbcb6e491fe814e06ea17a5617f565.pdf

Venables WN, Ripley BD (2002) Modern applied statistics with S. Springer, New York. ISBN 9780387954578

WHO (2003) World Health Organization/Unicef. Africa Malaria Report 2003. WHO/CDS/Mal/2003.1093. http://www.who.int/ malaria/publications/atoz/whocdsmal20031093/en/

WHO (2004) World Health Organization. A strategic framework for malaria prevention and control during pregnancy in the African region. In: A strategic framework for malaria prevention and control during pregnancy in the African region. http://www.afro.who.int/sites/default/files/2017-06/malaria_in_pregnancy_092004.pdf

WHO (2012) World Health Organization. Intermittent preventive treatment of malaria in pregnancy using sulfadoxinepyrimethamine. Geneva, World Health Organization. http://www.who.int/malaria/mpac/sep2012/iptp_sp_erg_meeti ng_report_july2012.pdf

WHO (2014) World Health Organization. A strategic framework for malaria prevention and control during pregnancy in the African region. In: a strategic framework for malaria prevention and control during pregnancy in the African region. https://www.mhtf.org/document/a-strategic-framework-for-malaria-prevention-and-control-during-pregnancy-in-theafrican-region

Yargawa J, Leonardi-Bee J (2015) Male involvement and maternal health outcomes: systematic review and meta- analysis. J Epidemiol Community Health 69(6):604-612. https://doi.org/10.1136/jech-2014-204784

\section{Submit your manuscript to a SpringerOpen ${ }^{\circ}$ journal and benefit from:}

- Convenient online submission

- Rigorous peer review

- Open access: articles freely available online

- High visibility within the field

Retaining the copyright to your article

Submit your next manuscript at $\gg$ springeropen.com 